\title{
Study of a possible detection of abnormalities under skin tissue by infrared thermography
}

\author{
by H. Trabelsi ${ }^{* * *}$, N. Elkadri* and E. Sediki ${ }^{* \star}$ \\ * Université de Tunis El Manar, Laboratoire de Biophysique et de Technologies Médicales, Institut \\ Supérieur des Technologies Médicales de Tunis, 9 Rue Zouhaier Essafi, 1006 Tunis, Tunisia \\ ** Université de Tunis El Manar, Unité de Rayonnement Thermique, Faculté des Sciences de Tunis, \\ 2092 El Manar I, Tunis, Tunisia
}

\begin{abstract}
This is a simulation study focusing on the effect of the temperature response of a biological tissue due to the existence of abnormal inclusions under skin or abnormal functionality. The Pennes bioheat transfer equation is used as a biophysical model to describe heat loss and circulatory system contribution to heat transfer in a tissue under skin. The objective of this study is the to obtain the temperature mapping on the skin, in view of the existence of abnormal inclusion or abnormal functionality, and later, see the influence of different parameters such as location, size, conductivity, metabolic generation heat and blood perfusion on the temperature mapping. After the introduction of the mathematical model of bioheat transfer in the skin tissue, we present the discrete grid of the medium and boundary conditions including radiation. Finally, we give numerical results concerning detection of abnormalities under skin tissue. We try to extend the obtained model to handle clinical observations concerning evaluation of patients at high risk for lower extremity peripheral arterial disease..
\end{abstract}

\section{Introduction}

The single-point temperature is used as an indicator for health in medical routine. However, it is possible that temperature maps at multiple points or thermograms can provide diagnostic information about anatomy and functionality of explored organs or tissues [1]. In fact, it is established that abnormalities affecting blood circulation, local metabolism, inflammation can affect skin temperature mapping. As a theoretical investigation, temperature mapping can be derived from a balance of heat transfer by conduction through the tissue, the metabolic heat production of tissue and the rate of volumetric blood. We can suppose that infusion is proportional to the gradient between arterial blood temperature and the temperature of the local tissue, where the blood temperature approached core temperature of the body. At the skin surface, the temperature is also affected by convection and radiation heat transfer with ambient air. The main objective of this work is to show the possibility of temperature mapping to predict whether location and size of imbedded abnormal inclusions or premature abnormal functionality into normal tissue. First, we analyze the Pennes model proposed to simulate the heat transfer in a human body. Second, we give a numerical implementation of the model in a bidimensional case. In particular, we analyze the linearization of radiative boundary conditions. Finally, we present some results about detection of tumor under skin tissue by thermography. We try to extend the obtained model to evaluate blood circulation in diabetic patients.

\section{Numerical simulation of tumor detection}

Bioheat transfer which is developed here is based on the Pennes equation [2]. This model supposes that the biological material is homogeneous and has isotropic thermal properties. The blood vessels are assumed to be isotropic. It suits for heat transfer in living tissue. It takes into account only metabolic heat generation and convective heat transfer due to the circulation of blood. In two-dimensional case, we consider a region formed of two sub-domains as shown in Figure 1. Sub-domain 1 is a rectangular Layer under the skin; it is formed by healthy tissue. The second sub-domain is a circular tumor formed by affected tissue. The corresponding mathematical expression of steady-state bio-heat balance is given through the following equation:

$$
\lambda_{\mathrm{i}}\left(\frac{\partial^{2} \mathrm{~T}_{\mathrm{i}}}{\partial \mathrm{x}^{2}}+\frac{\partial^{2} \mathrm{~T}_{\mathrm{i}}}{\partial \mathrm{y}^{2}}\right)+\mathrm{W}_{\mathrm{b}} \mathrm{C}_{\mathrm{b}}\left(\mathrm{T}_{\mathrm{b}}-\mathrm{T}_{\mathrm{i}}(\mathrm{x}, \mathrm{y})\right]+\mathrm{Q}_{\mathrm{m}_{\mathrm{i}}}=0
$$

where $\mathrm{i}=1,2$, is a number which is relative to healthy tissue and affected tissue (tumor) respectively, $T(x, y)$ is the tissue temperature, $\lambda$ is the tissue thermal conductivity, $W_{b}$ is the blood perfusion rate, $C_{b}$ is the blood specific heat, $T_{b}$ is the blood temperature, and $\mathrm{Q}_{\mathrm{m}}$ is the tissue metabolic heat generation rate. 
Results of numerical simulation allow to obtain the temperature mapping on the skin, in view of the existence of a tumor, and to see the influence of different parameters such as location, size, conductivity, metabolic generation heat and blood perfusion.

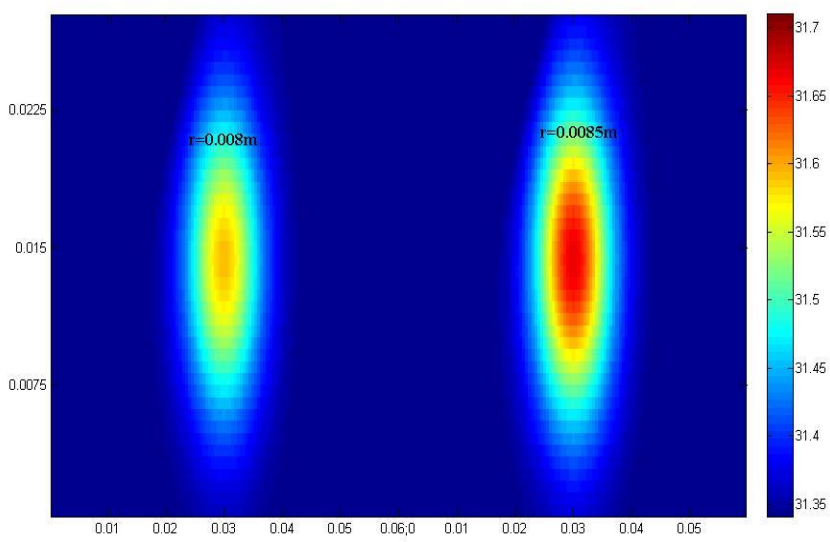

Fig. 1. Effect of tumour size on temperature mapping at skin surface

\section{Extension to early detection of vascular disease}

We tried to extend the obtained model to handle the possibility of early detection of peripheral vascular disease essentially for the patients with diabetes [3]. We compare numerical temperature mapping obtained by numerical simulation with thermograms of confirmed patients. This could evaluate blood circulation for high risk patients

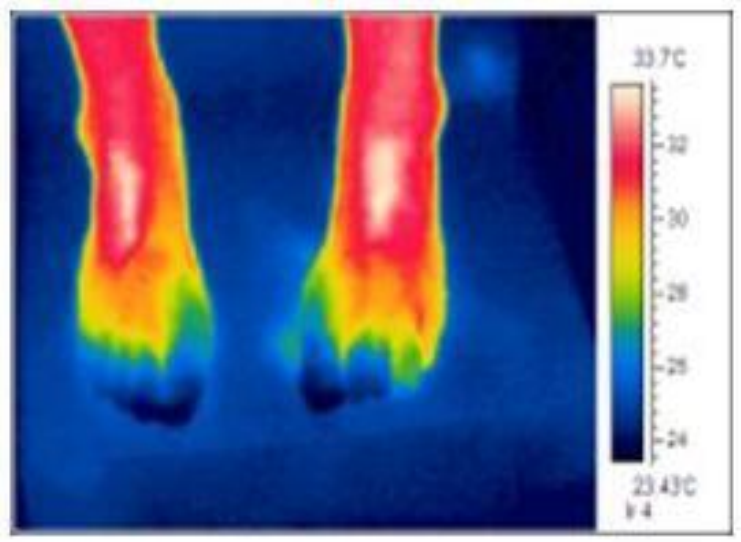

Fig. 2. Infrared thermal image of the feet of a patient with diabetes exposed to peripheral disease

\section{REFERENCES}

[1] D. Pascoe, , J.B Mercer, and De Weerd, L., Physiology of Thermal Signals. In: The Biomedical Engineering Handbook, 3rd. edition; Medical Devices and Systems, CRC press, New York chapter 21, pp 1-20, 2006

[2] J. P. AgnelliA. A. Barrea C. V. Turner. Tumor location and parameter estimation by thermography, Mathematical and Computer Modelling Volume 53, pp 1527-1534, 2011

[3] CL Huang, YW Wu , CL Hwang, YS Jong, CL Chao, WJ Chen, YT Wu, WS Yang. The application of infrared thermography in evaluation of patients at high risk for lower extremity peripheral arterial disease, $\underline{\mathrm{J}}$ Vasc Surg. 54(4), pp1074-80, 2011 